\title{
Patient-Reported Outcomes for Migraine in the US and Europe: Burden Associated with Multiple Preventive Treatment Failures
}

\author{
Janet Ford' \\ Russell M Nichols (D) \\ Wenyu Ye' \\ Antje Tockhorn- \\ Heidenreich (D) \\ Sarah Cotton (iD ${ }^{2}$ \\ James Jackson (iD ${ }^{2}$ \\ 'Eli Lilly and Company, Indianapolis, IN, \\ USA; ${ }^{2}$ Adelphi Real World, \\ Bollington, UK
}

Purpose: To evaluate patient-reported outcomes (PROs) among patients with migraine, including those who were preventive-naïve and preventive-treated.

Methods: This was a point-in-time, real-world study of patients with migraine in the US and EU5 (France, Germany, Spain, Italy, and UK) and their physicians using data from the Adelphi Migraine Disease Specific Programme (DSPTM). Physicians completed patient record forms (PRFs) for the next nine consulting patients with migraine plus a tenth patient, who did not need to be consecutive, for whom prior preventive migraine treatments had failed at least once, in order to achieve oversampling of such patients. Patients were given self-completion (PSC) forms that included the Migraine-Specific Quality of Life Questionnaire v2.1 (MSQ), Migraine Disability Assessment Scale (MIDAS), and Work Productivity and Activity Impairment (WPAI) questionnaire. Populations of interest included preventive-naïve and preventive-treated patients defined by the number of treatment lines (12 or $3+$ preventive regimens). Continuous variables were compared using $t$-test or ANOVA if normally distributed and Mann-Whitney if not. Chi-squared was used for categorical variables.

Results: During August-December 2017, 615 physicians (359 PCPs, 256 neurologists) completed PRFs for 5785 patients $(71 \%$ female; mean age $40( \pm 14)$ years; $65 \%$ in full- or part-time employment). Of these, 2798 completed a PSC (preventive-naïve/1-2/3+ preventive lines, $n=1707 / 1034 / 57$ ). Preventive-treated patients had a greater patient-reported burden across multiple measures versus preventive-naïve patients. Preventive-treated patients had lower MSQ scores indicating greater functional impairment, higher MIDAS scores indicating greater migraine-associated disability, and higher WPAI scores indicating greater overall work and activity impairment than preventive-naïve patients. The magnitude of difference was greatest for the $3+$ preventive-treatments cohort. Patterns were similar in the US and EU5.

Conclusion: Among patients with migraine who are preventive-treated, including those with multiple lines of therapy, there remain considerable unmet needs in terms of restoring patient function.

Keywords: migraine, preventive, patient-reported outcomes, real-world

\section{Introduction}

Migraine is a disabling primary headache disorder that is associated with a decline in health-related quality of life (HRQoL) as a result of the severity of the head pain, neurological symptoms, attack frequency, and interictal burden. ${ }^{1}$ Even moderate migraine attacks can disturb normal activities, and the loss of health or wellbeing is
Correspondence: Janet Ford

Eli Lilly and Company, Indianapolis, IN, 46285, USA

Tel + 317-903-8199

Email ford_janet@lilly.com 
felt both during and between migraine attacks. ${ }^{2}$ The global prevalence of migraine is $11.6 \%$ and is higher in females and in mid-life. ${ }^{3}$ With the prevalence of migraine peaking during prime employment ages, work productivity is compromised in affected individuals, including skills such as problem solving, and activities such as speaking and driving. ${ }^{4,5}$

Research suggests that the use of preventive medications that reduce the frequency of migraine can lead to improvements in $\mathrm{HRQoL}^{6}$ and reduction in migrainerelated disability. ${ }^{7}$ Nonpharmacologic interventions have also been found to be effective as preventative treatments, with comparative trials demonstrating an additive effect of the concomitant use of behavioral and pharmaceutical therapies. ${ }^{8}$ Persistence with preventive medications for migraine has been shown to be poor; specifically, analysis of a US claims database identified a persistence rate with oral preventive medications of $25 \%$ after 6 months and 14\% after 12 months. ${ }^{9}$ Patient cycling through different preventive medications has been observed: in the second International Burden of Migraine Study, the mean number of prophylactic medications ever used was 2.92 for patients with episodic migraine and 3.94 for those with chronic migraine. ${ }^{10}$ Common reasons for discontinuation of preventive treatments included adverse events and a lack of efficacy. ${ }^{10}$ Meaningful reductions in headache frequency can be a challenge if achieving adequate dosage and duration is not feasible as a result of patients being unable to tolerate their medication. ${ }^{9,10}$ Likewise, the treatment goal of improving patient functioning and decreasing disability is also difficult to attain. ${ }^{11-13}$

Inadequately managed migraine has been shown to adversely impact patients' functioning, leading to reduced productivity, restricted family and social activities, and an overall decline in HRQoL. ${ }^{14,15}$ Patient-reported migraine outcomes among those with various preventive treatment lines, including preventive-naïve (no migraine preventive medications), have not been widely studied across multiple countries. The primary aim of this research study was to evaluate disease burden differences across three groups as defined by the number of preventive treatment lines $(0$ lines [preventive-naïve], 1-2 lines, and 3+ lines) using data from the Adelphi Migraine Disease Specific Programme (DSPTM), a point-in-time survey of physicians and their consulting patients with migraine in the US and EU. The disease burden outcomes evaluated included migraine-associated disability, functional impairment, general health state, and work productivity. Demographic and clinical characteristics were also analyzed.

\section{Materials and Methods Study Design}

This was a point-in-time study characterizing patients with migraine in the US and the EU5 (France, Germany, Spain, Italy, and UK). Data were drawn from the Adelphi Migraine DSP, which comprised primary care physicians (PCPs), neurologists (including headache specialists), and their patients. The DSP methodology has been described in detail elsewhere. ${ }^{16-18}$

The DSP methodology and questionnaires were reviewed by an official independent body, Freiburger Ethik-Kommission International (FEKI), which granted ethical approval. DSP data were collected in accordance with Adelphi Real World procedures, which are compliant with the Health Information Technology for Economic and Clinical Health (HITECH) Act, the Health Insurance Portability and Accountability Act (HIPAA), and the European Pharmaceutical Market Research Association (EphMRA). The study was conducted in accordance with the Declaration of Helsinki. All patients provided written informed consent to participate after receiving details of the study.

\section{Study Population}

Physicians were identified and recruited through networks of professional recruiters, using public and internal databases; measures were taken to ensure a geographical spread across the regions. Physicians were required to be PCPs or neurologists working in any type of setting. PCPs had to be consulting with $\geq 10$ patients with migraine in a typical month and neurologists had to be consulting with $\geq 20$ patients with migraine in a typical month; both physician types were actively involved in the treatment and management of patients with migraine.

Physicians recruited the next nine consecutive patients with migraine who were attending for a consultation (consulting patients) and completed a detailed patient record form (PRF) for each patient. A tenth patient was recruited, for whom prior preventive treatments for migraine (eg anticonvulsants, beta-blockers, antidepressants, calcium channel blockers, and others) had failed at least once, in order to achieve oversampling of such patients; this patient did not need to be consecutive. The populations of interest in this study included preventive-naïve and preventive- 
treated patients, defined by the number of migraine preventive pharmacological treatment lines received (1-2 or 3 + preventive regimens).

The PRF was used to collect demographic and clinical information on the patient, including a detailed treatment history. Physicians were compensated for participating in the DSP according to fair market research rates consistent with their time involved.

To be eligible for inclusion in the study, patients had to be aged $\geq 18$ years, with a diagnosis of migraine and actively consulting a participating physician. No other formal patient selection verification procedures were used. Eligible patients were asked to complete a patient self-completion form (PSC). Only patients for whom a PRF was provided were invited to complete a PSC, allowing data provided by the patients to be linked to data provided by the physician in the PRF. The PSC contained detailed questions on demographics and medication use. Patients also completed a number of validated questionnaires relating to the physical and emotional impact of their disease: Migraine-Specific Quality of Life Questionnaire (MSQ; version 2.1), ${ }^{19}$ Migraine Disability Assessment Scale (MIDAS), ${ }^{20,21}$ EQ-5D-5L, ${ }^{22,23}$ and Work Productivity and Activity Impairment (WPAI) questionnaire. ${ }^{24}$ Patients were asked to complete the PSC away from and without the assistance of the physician. Completing the PSC was voluntary and in cases where the patient decided not to complete the questionnaire, physician-recorded data, but not patient-reported outcome (PRO) data, for that patient could still be included in the analysis.

\section{Outcomes}

The MSQ measures the effect of migraine and its treatment on patient HRQoL over three dimensions: how migraines restrict daily social and work-related activities (Role Function-Restrictive); how migraines prevent these activities (Role Function-Preventive); and the emotional impact of these migraines (Emotional Function). Response options range from 1 (none of the time) to 6 (all the time). After computation of the raw score for a domain (or the total), the score is linearly transformed to a $0-100$ scale, with 100 indicating full functionality.

The MIDAS was designed to measure and quantify disability related to headache over a 3-month period. MIDAS consists of five items that reflect the number of days reported as missing or with reduced productivity at work or home and social events because of headache.
Disability scores are defined as follows: $0-5=$ little or no disability, $6-10=$ mild disability, $11-20=$ moderate disability, $21-40=$ severe disability, and $\geq 41=$ very severe disability.

The EQ-5D-5L consists of two parts: the EQ-5D-5L descriptive system and the EQ visual analog scale (VAS). The descriptive system measures five dimensions: mobility, self-care, usual activities, pain/discomfort, and anxiety/depression. EQ-5D-5L utility scores were calculated using country-specific algorithms, alongside VAS scores. The utility score is defined as the numeric index of each patient population's health state; the VAS score is defined as the average numeric rating of the VAS score, with 0 corresponding to the worst imaginable health state and 100 to the best imaginable health state. In line with the National Institute for Health and Care Excellence guidance, EQ-5D-5L scores were cross-walked to $3 \mathrm{~L}$ scores. $^{25,26}$

The WPAI:Migraine questionnaire assesses migrainerelated work productivity and activity impairment. Four scores are calculated: absenteeism (work time missed), presenteeism (reduced effectiveness while at work), overall work impairment, and activity impairment. Each score ranges from 0 to $100 \%$ after transformation, with higher scores indicating greater impairment.

\section{Statistical Analysis}

These analyses were based on patients with a full preventive-treatment history. Preventive-naïve patients were identified as having no current or past history of preventive migraine pharmacological treatment. Preventive-treated patients were defined according to the number of regimens they had received (1-2 or $3+$ preventive regimens) for overall samples and by region (US and EU5).

Continuous variables were described by their frequency, mean, and standard deviation. Categorical variables were described by their frequency and percentage. Comparisons were made across three groups, preventivenaïve, 1-2 preventive lines, and $3+$ preventive lines of therapy.

To evaluate the differences between preventive-naïve patients and those who received 1-2 or $3+$ preventive lines of therapy, continuous variables with an approximately normal distribution were compared using a $t$-test or ANOVA and those not normally distributed were compared using a Mann-Whitney test. Categorical variables were compared using a chi-squared test. The ShapiroWilk test was used to test normality. 
In an additional analysis (Supplementary Methods), preventive-treated subpopulations were defined broadly to narrowly by the number of treatment lines $(1+, 2+$, and $3+)$ in order to understand how patient burden changed when a subpopulation is expanded or restricted (1+ to $3+$ failures). In this analysis, the preventive-treated subpopulations were not mutually exclusive; that is, the $3+$ preventive lines group was a subpopulation of the $2+$ and $1+$ preventive lines groups, and the $2+$ preventive lines group was a subpopulation of the $1+$ preventive lines group.

To evaluate the differences between preventive-naïve patients and those who received $1+, 2+$, or $3+$ preventive lines of therapy, continuous variables with an approximately normal distribution were compared using a $t$-test, and those not normally distributed and ordinal (ordered categorical) were compared using a Mann-Whitney test. The Shapiro-Wilk test was used to test normality; numerical variables that failed the Shapiro-Wilk normality test were compared using a Mann-Whitney test.

Stata version 15.1 or later was used to run the analyses. All statistical tests were performed at a two-sided 5\% significance level; $P$-values $<0.05$ were considered statistically significant.

\section{Results}

\section{Patients and Physicians}

Between August and December 2017, 615 physicians (152 in the US; 463 in the EU5), comprising 359 PCPs (101 in the US; 258 in the EU5) and 256 neurologists (51 in the US; 205 in the EU5), completed PRFs for 6057 patients (1519 in the US; 4538 in the EU5), 3523 of whom were treated by PCPs and 2534 treated by neurologists. A total of 5785 patients (1418 in the US; 4367 in the EU5) had a full preventive-treatment history. A PSC was completed by 2922 patients ( 885 in the US; 2037 in the EU5), of whom 2798 (827 in the US; 1971 in the EU5) had a full preventive-treatment history and comprised the PRO analysis cohort.

Demographic characteristics of the 5785 patients with full preventive-treatment history are summarized in Table 1. Most patients were female $(\mathrm{n}=4128 ; 71 \%)$ and of working age (mean age 40 years); two-thirds $(65 \%)$ were in full-time or part-time employment. Statistically significant differences were seen across the treatment categories in age and employment status, as shown in Table 1. In both the US and the EU5, a higher proportion of patients with three or more lines of preventive treatment were on long-term sick leave than preventive-naïve patients or those with 1-2 treatment lines. Preventivetreated patients were also more likely to have had to reduce their hours worked due to migraine than preventive-naïve patients (both $P<0.001$ across groups).

The clinical characteristics of patients are shown in Table 2. Overall, migraine without aura was the most common diagnosis; unilateral pain was the most troublesome symptom overall. Statistically significant differences between patient groups were observed, including migraine with aura diagnosis. The frequency of migraine headache days per month increased across treatment categories in the US and EU (both $P<0.0001$ ): $19 \%$ of US patients and $15 \%$ of EU5 patients who had $3+$ lines of preventive treatment had 15 or more migraine headache days per month, which was a 2-3 times greater proportion than the other treatment groups. Comorbid pain conditions were present in $18 \%$ of US patients and $13 \%$ of those in the EU5. Anxiety and depression, two of the most commonly reported comorbidities, differed significantly according to the number of treatment lines, being more prevalent in the preventive-treated subpopulations than preventive-naïve patients (all $P<0.0001$ ).

\section{Patient-Reported Outcomes}

A clear and statistically significant pattern in MSQ scores was observed across all domains, treatment groups, and regions, with lower scores, indicating greater functional impairment, in preventive-treated patients compared with preventive-naïve patients (Figure 1).

A greater proportion of patients with $3+$ lines of preventive treatment had moderate to very severe disability on the MIDAS scale compared with preventive-naïve patients and those with 1-2 lines of preventive treatment (Figure 2). Notably, among patients with $3+$ lines of preventive treatment, $35 \%$ of US patients and $33 \%$ of those in the EU5 had scores $\geq 41$, which is classified as having a very severe disability, compared with only $1 \%$ and $2 \%$, respectively, of preventive-naïve patients in the US and EU5. MIDAS Total and individual item scores are shown in Table 3.

Mean EQ-5D-5L utility and VAS scores are shown in Figure 3. In both regions, scores were statistically significantly different across the treatment groups, being lower in preventive-treated versus -naïve patients. Overall utility and VAS scores in patients who were preventive-naïve and those who had 1-2 


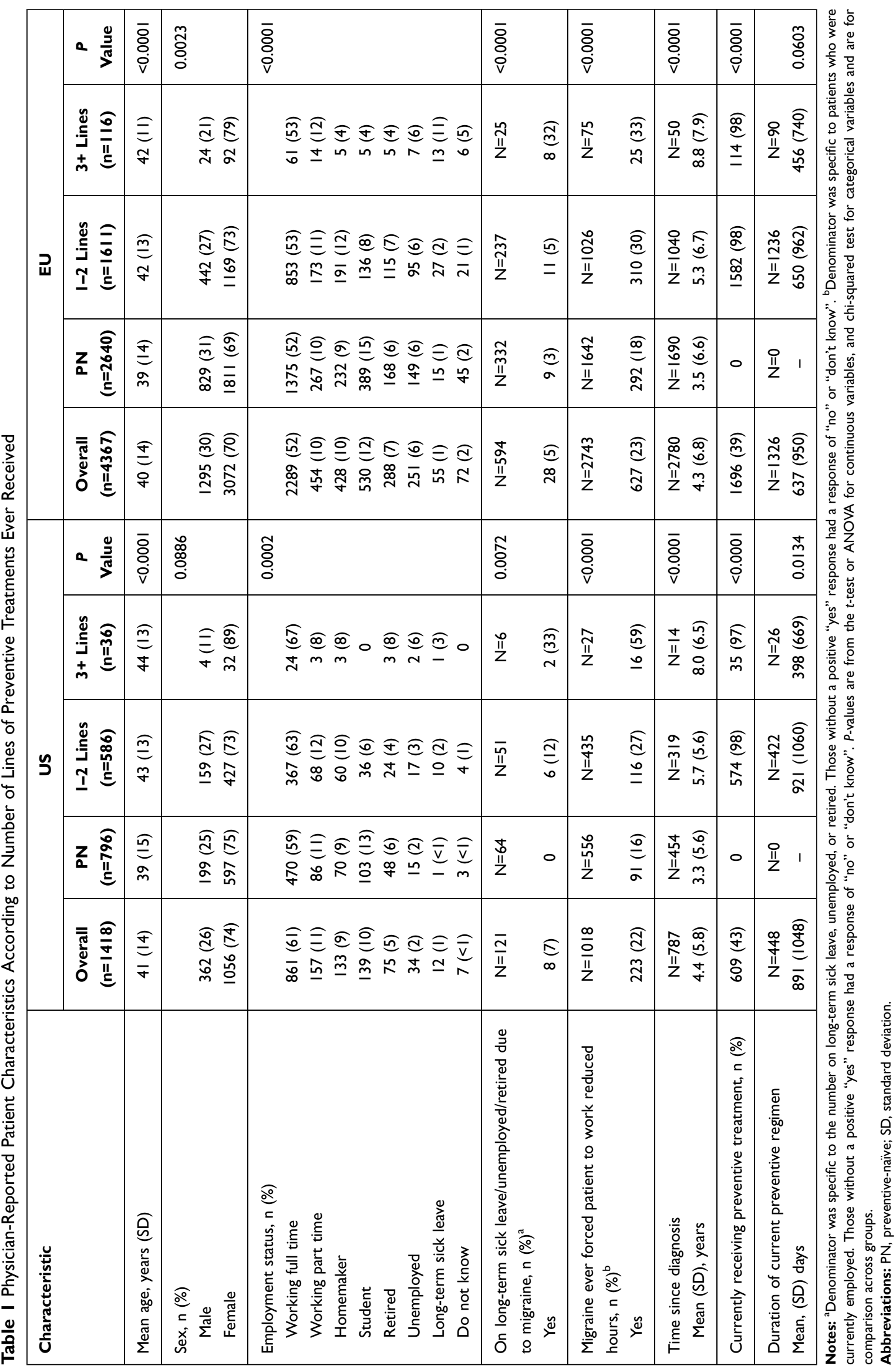




\begin{tabular}{|c|c|c|c|c|c|c|c|}
\hline \multirow{5}{*}{ כ } & $a \frac{\frac{0}{J}}{\frac{N}{\nu}}$ & 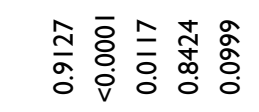 & $\begin{array}{l}\bar{o} \\
\dot{0} \\
\dot{v}\end{array}$ & $\begin{array}{l}\overline{8} \\
\text { ठ } \\
\text { v }\end{array}$ & 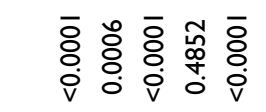 & 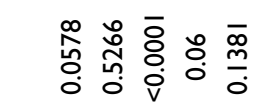 & 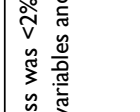 \\
\hline & 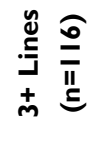 & 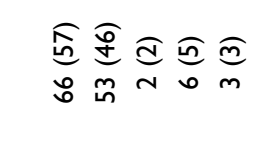 & 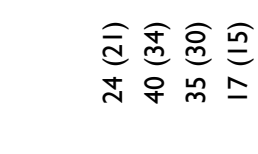 & 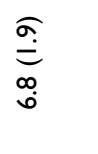 & 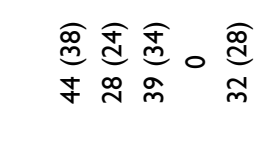 & 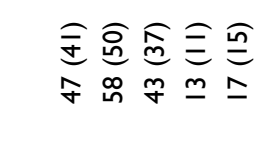 & 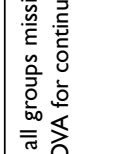 \\
\hline & 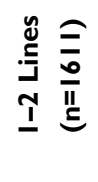 & 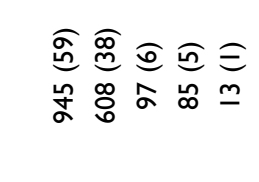 & 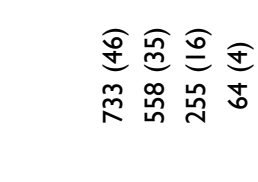 & 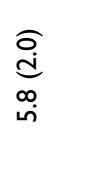 & 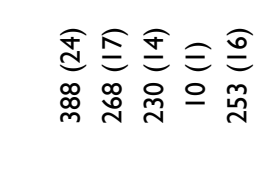 & 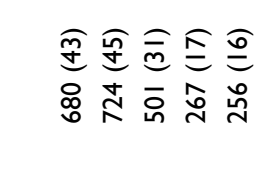 & 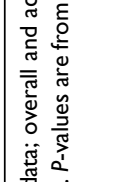 \\
\hline & $z \underset{a}{\stackrel{o}{+}}$ & 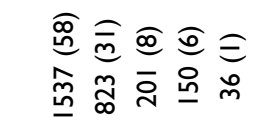 & 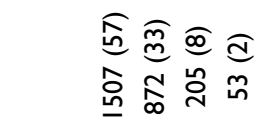 & 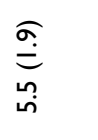 & 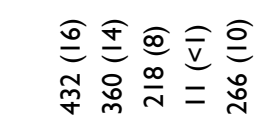 & 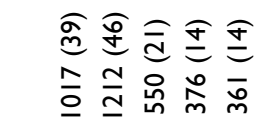 & 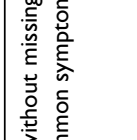 \\
\hline & 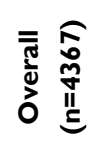 & 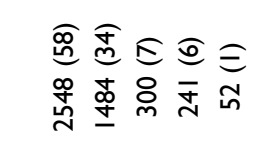 & 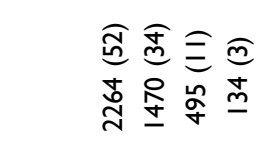 & $\begin{array}{l}\stackrel{\widehat{o}}{\mathrm{~d}} \\
\stackrel{0}{\circ} \\
\text { in }\end{array}$ & 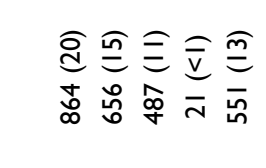 & 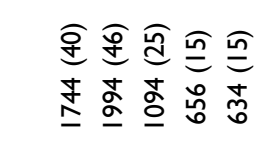 & 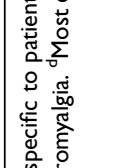 \\
\hline \multirow{5}{*}{$\stackrel{n}{د}$} & $a \frac{\frac{0}{3}}{\frac{\pi}{J}}$ & 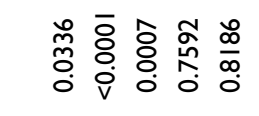 & $\begin{array}{l}\overline{8} \\
\text { ¿ } \\
\text { v }\end{array}$ & 商 & 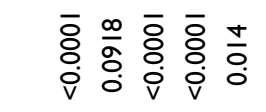 & 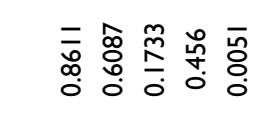 & है के \\
\hline & 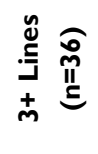 & 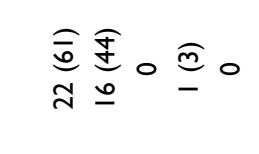 & 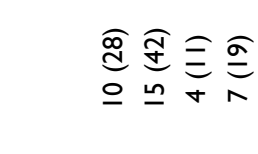 & $\begin{array}{l}\text { ָู } \\
\text { un } \\
\text { un }\end{array}$ & 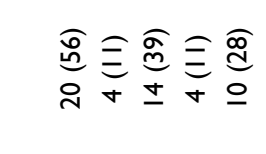 & 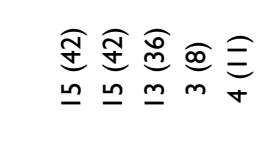 & 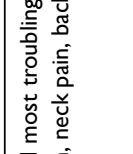 \\
\hline & 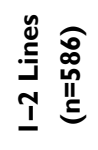 & 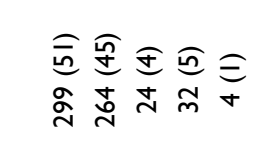 & 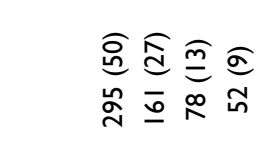 & 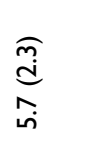 & 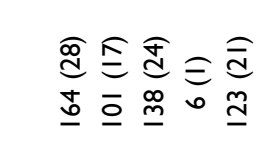 & 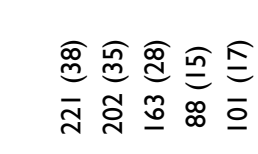 & 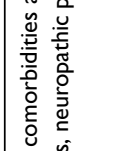 \\
\hline & $z \underset{0}{\stackrel{\varrho}{\circ}}$ & 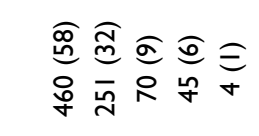 & 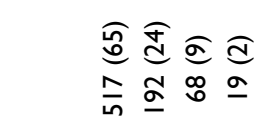 & $\begin{array}{l}\stackrel{\widehat{o}}{\mathrm{~d}} \\
\stackrel{0}{\circ}\end{array}$ & 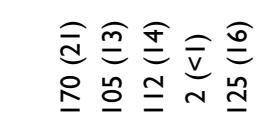 & 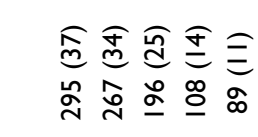 & 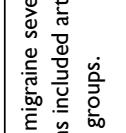 \\
\hline & o & 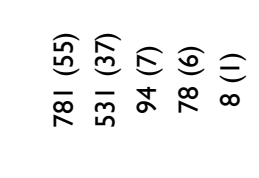 & 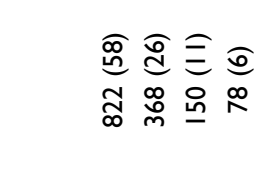 & $\begin{array}{l}\widehat{\bar{d}} \\
\text { hi }\end{array}$ & 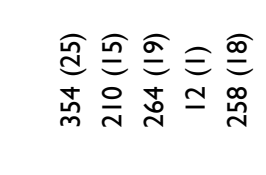 & 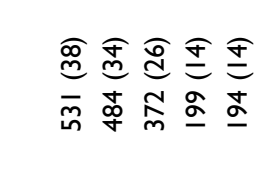 & 总 \\
\hline \multicolumn{2}{|l|}{ 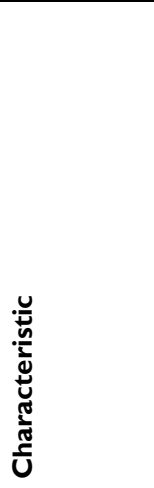 } & 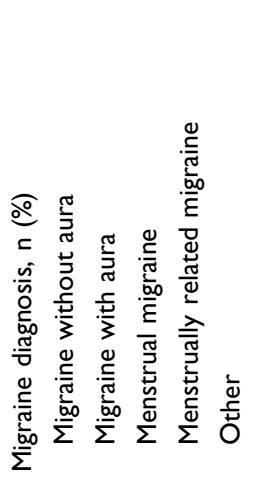 & 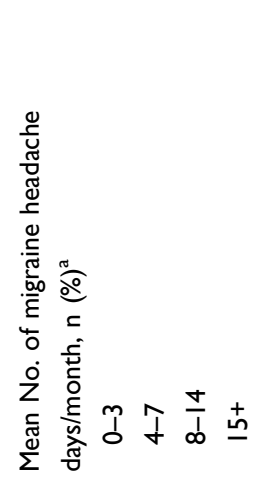 & 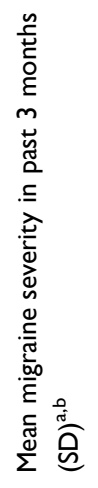 & 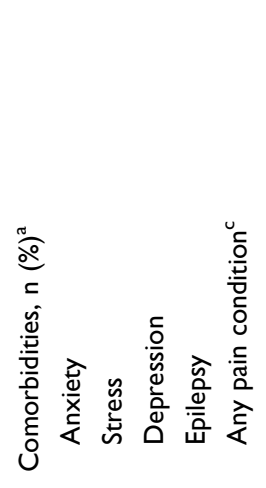 & 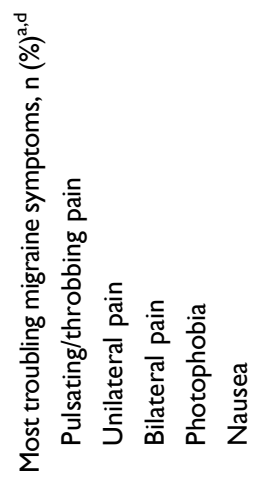 & \\
\hline
\end{tabular}




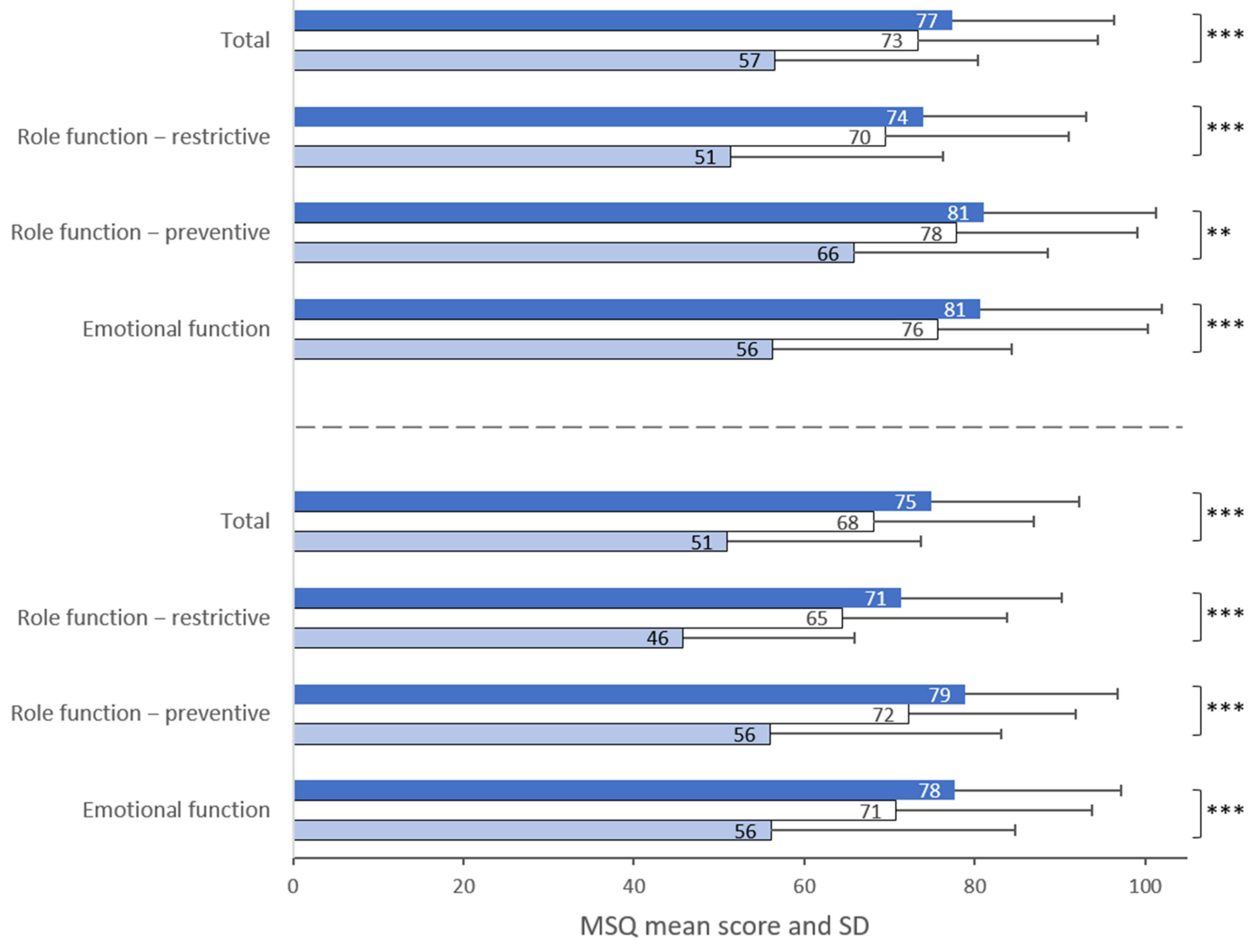

US

@ Preventive naïve $\square$ 1-2 lines $\square 3+$ lines

Figure I Migraine-Specific Quality of Life Questionnaire (MSQ) domain scores according to number of lines of preventive treatments ever received. The number of patients with data varied by group size (see Table I). Across all three groups, the response rate for this instrument was $98 \%$ ( 2748 of 2798 ) of patients who completed a patient self-completion form. Notes: $* * * P<0.0001$ across groups; $* * P<0.01$ (ANOVA test). Higher scores indicate better health status. Trends were consistent across regions (US $+E U)$.

Abbreviation: SD, standard deviation.

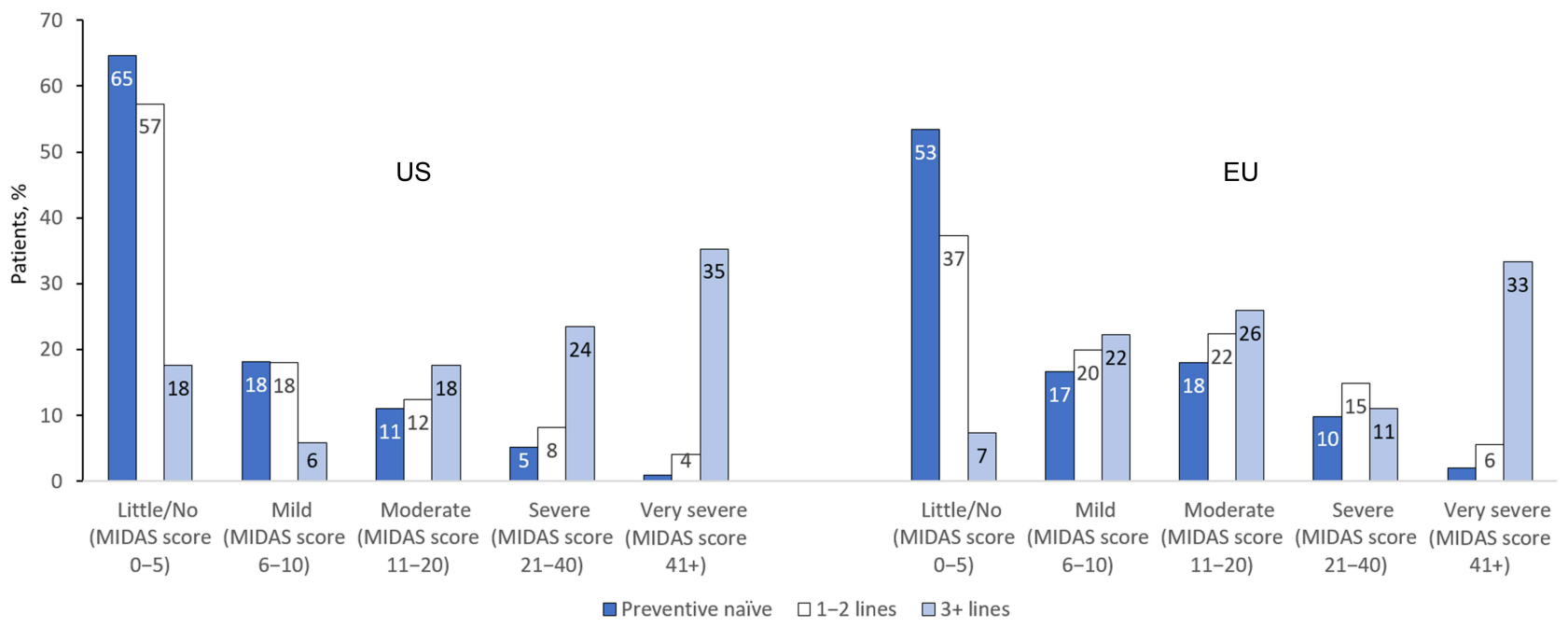

Figure 2 Migraine Disability Assessment Scale (MIDAS) scores according to number of lines of preventive treatments ever received. Higher scores indicate greater disability. The number of patients with data varied by group size (see Table I). Across all groups, the response rate for this instrument was $83 \%$ ( 23 I 6 of 2798 ) of patients who completed a patient self-completion form. Trends were consistent across regions (US + EU).

Notes: $P<0.000 \mathrm{I}$ across all treatment groups and all severity categories (chi-squared test). 


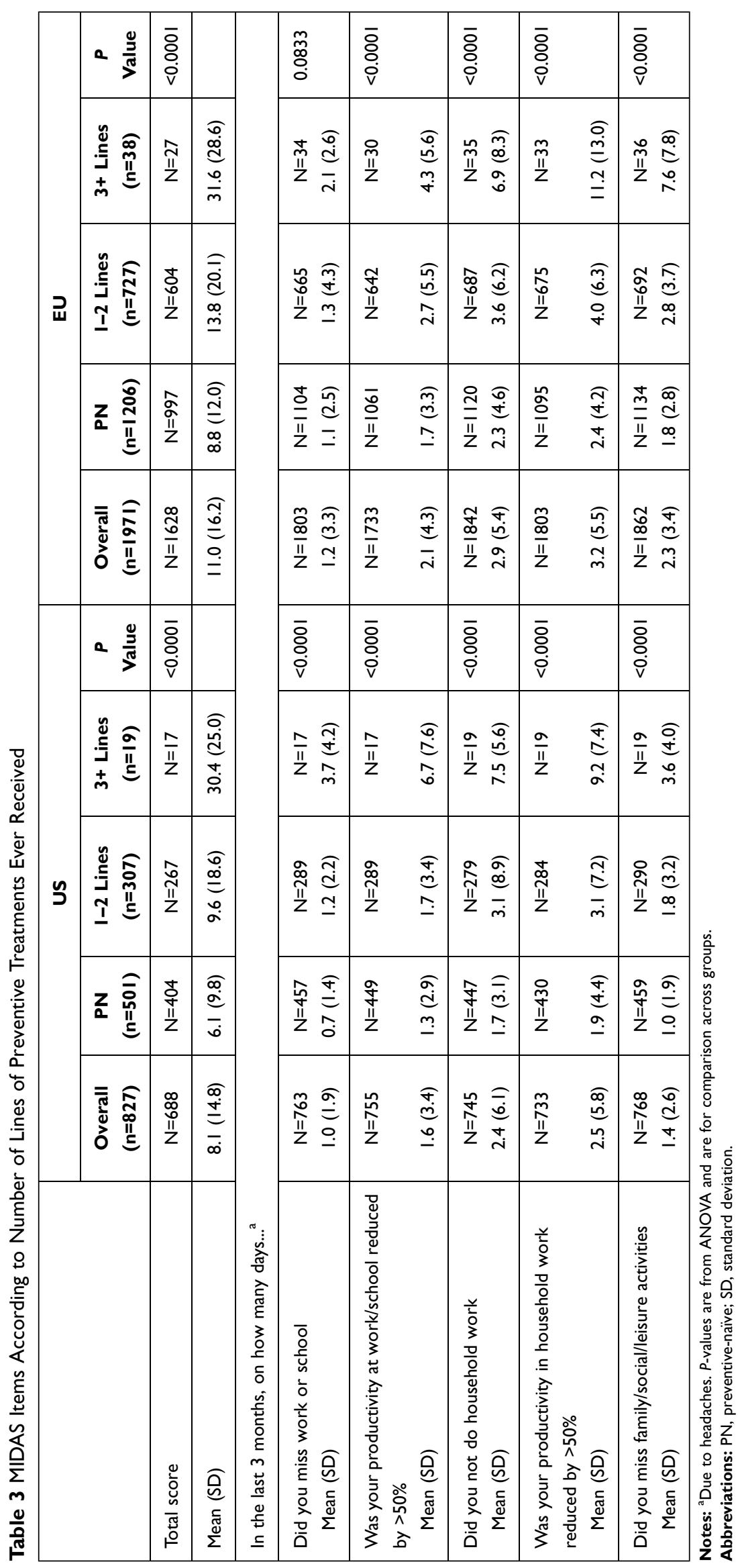



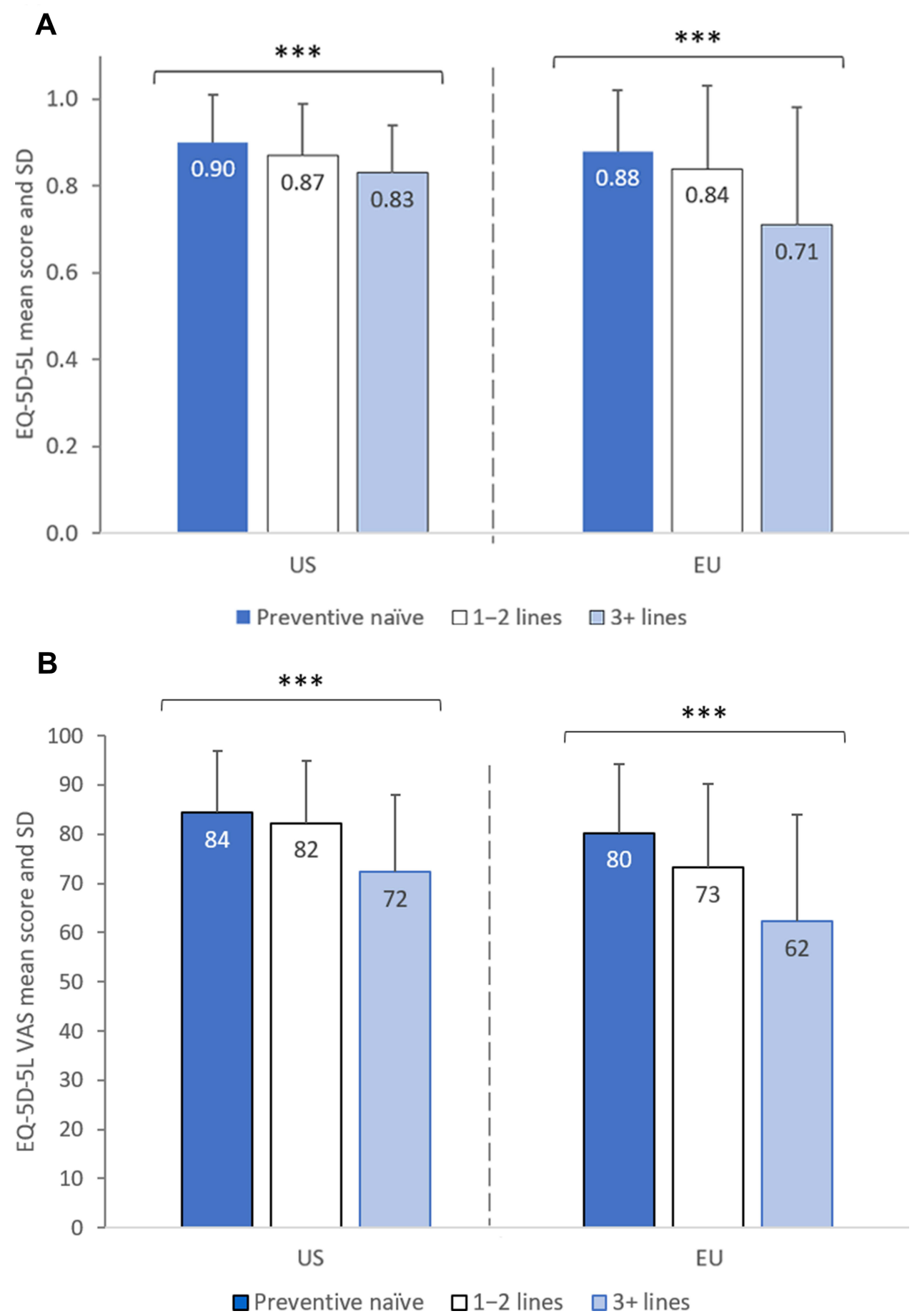

Figure 3 EQ-5D-5L scores according to number of lines of migraine preventive treatments (A) mean EQ-5D 5-level score cross-walked to 3-level score and (B) mean VAS score. Patients completed the 5-level EQ-5D; scores were cross-walked to the 3-level version. ${ }^{25,26}$ The number of patients with data varied by group size; the response rate for this instrument was $99 \%$ ( 2763 of 2798 patients who completed a patient self-completion form).

Notes: ***P<0.000I across groups (ANOVA test).

Abbreviations: EQ-5D-5L, EuroQol 5-Level 5-Dimension questionnaire; SD, standard deviation; VAS, visual analog scale.

preventive lines were similar to general population norms; EU patients with $3+$ preventive lines had lower scores than the general population, whereas those in the US had similar scores to the general population. $^{27}$
The number of patients with WPAI data varied by group size; the response rate for this instrument was $54 \%$ for work productivity impairment (patients in employment only; 1440 of 2798 patients who completed a PSC) and $94 \%$ for activity impairment (2633 of 2798 patients who 


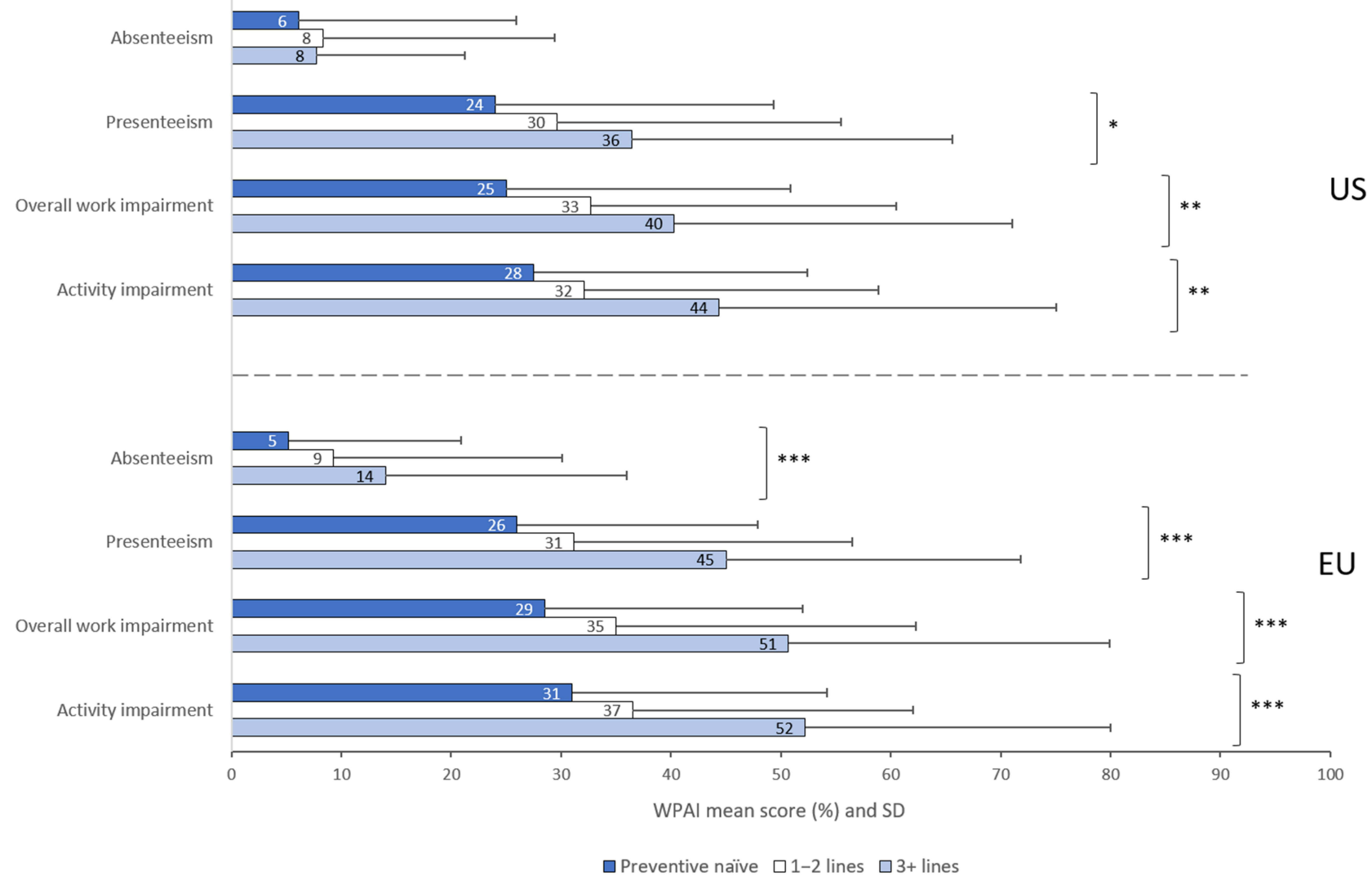

Figure $4 \mathrm{WPAl}$ in the overall patient population according to number of lines of migraine preventive treatments. The number of patients with data varied by group size; the response rate for this instrument was $54 \%$ for work productivity impairment (patients in employment only; 1440 of 2798 patients who completed a PSC) and $94 \%$ for activity impairment (2633 of 2798 patients who completed a patient self-completion form).

Notes: $* * * P<0.001 ; * * P<0.01 ; * P<0.05$ across groups (ANOVA test).

Abbreviations: SD, standard deviation; WPAI, Work Productivity and Activity Impairment.

completed a PSC). The number of lines of preventive treatment for migraine had a significant association with the patient's ability to perform at work and undertake normal activities as measured using the WPAI (Figure 4). WPAI scores were generally significantly higher, indicating greater work productivity and activity impairment, in preventive-treated versus preventive-naïve patients for all categories and in both regions (all $P<0.05$ ). One exception was absenteeism, which did not differ significantly across groups in the US.

Results of the additional analysis (Supplementary

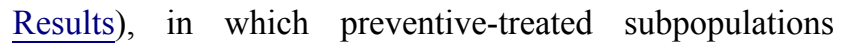
were defined broadly to narrowly by the number of treatment lines $(1+, 2+$, and $3+)$, are shown in Supplementary Figures $1-4$. In general, patients in the $3+$ preventive lines subgroups had a greater migraine burden than those with more broadly defined preventive-treated migraine and those who were preventive-naïve.

Scatter plots for these PRO outcomes, showing the spread of the data, are shown in Supplementary Figure 5.

\section{Discussion}

This descriptive analysis of real-world PRO data from a consulting migraine population in the US and EU5 has highlighted differences between patients prescribed preventive medications (available in 2017) for their disease compared with those who were preventive-naïve. Statistically significant differences across categories of patients, namely those who were preventive-naïve, those who had one or two preventive medications, and those who had received three or more preventive treatments, were observed in migraine-related disability, functional impairment, and reduced work productivity. In addition, the EQ-5D health utility and VAS scores were statistically significantly different across groups, with lower scores, indicating poorer health status, in the preventive-treated subpopulations than in preventive-naïve patients. These three categories also differed in their demographic and clinical characteristics; preventive-treated patients were more likely to be on long-term sick leave, unemployed, or retired due to migraine; and a diagnosis of migraine 
with aura was more prevalent among these patients compared with preventive-naïve patients. High disease burden was reported consistently across multiple PROs. This may have been influenced, at least in part, by comorbid pain conditions in subgroups of patients, which may have impacted on their reporting of outcome measures. Comorbidity with other pain conditions was common in the patient population, particularly in the more heavily pre-treated subgroup. Other pain conditions, which are frequent among patients with migraine, ${ }^{28}$ have been reported to both influence and be influenced by migraine, suggesting a complex relationship between pain conditions. $^{29,30}$ There is therefore a need to comprehensively evaluate and treat coexisting pain conditions in patients with migraine. ${ }^{30}$ These results indicate considerable unmet needs in patients with migraine who are being actively treated, including those cycling through different lines of preventive therapy.

These research findings are an important contribution to understanding the differences between preventive-naïve and preventive-treated migraine populations. Given the lack of global research specific to patients with migraine who are undergoing active treatment in the healthcare system, this study provides important real-world evidence on this topic. Notably, significant differences in HRQoL and disability were observed across groups, with the greatest impact of disease in patients with the highest number of preventive lines. Other research has shown an association between cycling through preventive treatment and headache frequency and comorbidities; ${ }^{31}$ the present extensive analyses of PRO measures provide complementary insight into the effects of having failed previous treatment lines in patients with migraine. The full complexity of these findings is unknown, as it relates to migraine disease severity, adverse effects of treatment regimens, the sequence of medical/treatment/life events, and other physical/psychological factors that likely play a role in these observed outcomes. This evidence further emphasizes the need to identify preventive care that works for patients with migraine, in particular before this neurological disease progresses to a refractory state and/or substantial patient burden is incurred. ${ }^{32}$ This reinforces the clinical practice of collecting a patient's treatment history, specifically the number of preventive lines received, and their current level of burden due to migraine disease, so that further intervention strategies, including non-pharmacological approaches, can be discussed with the patient when appropriate. This practice is increasingly important as new preventive treatments emerge and are addressed in treatment guidelines. ${ }^{32,33}$

Preventive-treated patients in this study were more likely than those who were preventive-naïve to have experienced an impact of the disease on their ability to work, as reflected by the proportion of patients who had been forced to work reduced hours because of migraine. For those who were employed, WPAI data indicated a statistically significantly higher proportion of absenteeism, presenteeism, overall work impairment, and activity impairment due to migraine in the EU population. A similar pattern was noted in the US population, with the exception of absenteeism, which did not differ statistically across the patient groups. This is perhaps in line with research suggesting that patients with migraine often attempt to continue working, despite their migraine attacks, in lieu of being absent from work. ${ }^{34}$ Differences across countries in the rights of employees to paid sick leave may also have influenced this finding. A report by the World Policy Analysis Center found that the US and South Korea were the only Organisation for Economic Cooperation and Development countries that do not guarantee paid leave for personal illness. ${ }^{35}$ Others have reported an increasing impact of migraine on WPAI scores in line with the frequency of migraine attacks, with the greatest effect being experienced by patients with chronic migraine. $^{36}$ Our findings support those of the My Migraine Voice study, which reported a greater impact of migraine on work productivity and daily activities as the number of treatment cycles increased. ${ }^{15}$ These data indicate a considerable societal impact of migraine in patients who did not respond to preventive therapies.

The MSQ data provided by our patients indicated that migraine restricted and prevented patient daily activities to a greater extent among the preventive-treated subpopulations compared with preventive-naïve patients. Other global research has demonstrated a relationship between cycling through preventive treatments and reports of lower HRQoL with a negative impact on functioning in patients with migraine. ${ }^{15}$ When evaluating disability associated with migraine, prior research in patients in France, Germany, Japan, and the US found that disability remained high whether patients were treated with preventive agents (available at that time) or not, possibly indicating the need for more effective migraine preventive agents. ${ }^{37,38}$ In the present study, disability was greater among the preventivetreated subpopulations than those who were preventivenaïve; further investigation is needed to determine barriers 
to achieving the treatment goal of improving patient functioning, including utilization of behavioral therapies. ${ }^{8}$

In regard to general health, mean EQ-5D VAS scores of 84 and 80 for preventive-naïve patients in the US and EU5, respectively, in the present study were comparable with population norms for people in the US (80.0) and Europe $(75.0-82.8) ;{ }^{27}$ however, VAS scores in patients with three or more lines of preventive therapy were notably lower than these population norms (72.3 in the US, and 62.3 in the EU5). In the US, the mean EQ-5D utility score of $0.83,0.87$, and 0.90 for patients with $3+$ preventive lines, patients with 1-2 lines, and preventive-naïve patients, respectively, were comparable with population norms $(0.83),{ }^{23}$ whereas in the EU5, the mean utility score of 0.71 for patients with $3+$ preventive lines was lower than EU population norms (0.86-0.92). These findings are in line with data reported by Shah et al, in which preventive-naïve patients had EQ-5D scores of 0.81-0.90 depending on the number of headache days experienced and those using preventive medications had scores of $0.70-0.88 .^{38}$ In that study, EQ-5D scores were found to be associated with the number of headache days, comorbidities, anxiety, depression, and country. ${ }^{38}$

The EQ-5D is subject to some limitations that should be considered. Generic tools are usually less sensitive than disease-specific outcomes measures and therefore less likely to capture differences between patient groups, such as those included in this analysis. The lack of sensitivity of the EQ-5D in this study may also be a consequence of how the data were collected in this point-in-time study. As patients were consulting their physician when invited to participate in the study, they were unlikely to be experiencing a migraine at that particular time and may have scored their condition higher than might be expected. These findings underline the complexity of measuring HRQoL in patients with migraine and the number of factors that should be considered in their treatment.

Some additional limitations of this analysis should be considered. In order to include a wide range of physicians and patients with varying treatment types and disease stages, the Adelphi Migraine DSP had few inclusion criteria other than patients having to be aged $\geq 18$ years with a diagnosis of migraine. Our study findings may not be generalizable to the entire migraine population; however, they are representative of patients diagnosed with migraine who are seeking care, which is approximately $20-40 \%$ of European patients with migraine and $41 \%$ of those in the USA. ${ }^{39,40}$ Frequent consulters may be over- represented in this study. Physician and patient participation was influenced by practical considerations of geographic location and willingness to participate. As the convenience sampling techniques used may have introduced selection bias, efforts were made to reduce this, including having physicians provide data for a consecutive series of patients, therefore minimizing this bias. However, the oversampled patient, who was included to boost sample size, may not have been consecutive. Even with the efforts made to recruit more patients with a history of at least one prior preventive treatment failure, the subgroup with $3+$ treatment failures was much smaller than the other subgroups; this imbalance in sample size may have implications for the reported effect sizes. These are point-in-time data, captured from the perspective of physicians and patients. Consequently, causality and a temporal relationship cannot be established between preventive treatment and outcomes as this would require longitudinal evaluation; this should be considered when comparing with other data sources. Patients may have cycled through treatments for varying reasons, including lack of efficacy and treatment side effects. Further research is needed to identify possible relationships between these factors and PRO results. Finally, this analysis is descriptive in nature and did not adjust for bias or confounding. The analytical techniques used were meant to preserve the full implications of what patients with migraine were reporting/experiencing, without controlling for other variables, as observed in realworld consulting patients. Unadjusted PRO data may be influenced by patient characteristics and caution should be taken when interpreting the results. Future research into the implications for outcomes of patient demographics and other variables is warranted.

\section{Conclusions}

In conclusion, this study of PRO data from real-world consulting patients with migraine in the US and EU5 suggests that the burden of migraine is greater among preventive-treated subpopulations when compared with preventive-naïve patients. Specifically, the magnitude of difference was greatest in patients cycling through multiple lines of preventive therapy. Patterns of migrainerelated disability, HRQoL impact, and work productivity impairment were generally consistent across the US and EU5. 


\section{Abbreviations}

DSP, Disease Specific Programme; EphMRA, European Pharmaceutical Market Research Association; FEKI, Freiburger Ethik-Kommission International; HIPAA, Health Insurance Portability and Accountability Act; HITECH, Health Information Technology for Economic and Clinical Health; HRQoL, health-related quality of life; MIDAS, Migraine Disability Assessment Scale; MSQ, Migraine-Specific Quality of Life Questionnaire; PCP, primary care physician; PRF, patient record form; PRO, patient-reported outcome; PSC, patient self-completion; VAS, visual analog scale; WPAI, Work Productivity and Activity Impairment.

\section{Data Sharing Statement}

The data that support the findings of this study are available from Adelphi Real World, but restrictions apply to the availability of these data, which were used under license for the current study and so are not publicly available.

\section{Ethical Approval}

The study was approved by an official independent body Freiburger Ethik-Kommission International (FEKI). Informed consent was obtained from all individual participants included in the study.

\section{Acknowledgments}

Medical writing assistance was provided by Deirdre Carman and funded by Eli Lilly and Company. The first draft of the manuscript was written by Deirdre Carman and all authors commented on previous versions of the manuscript.

\section{Author Contributions}

All authors made substantial contributions to conception and design, acquisition of data, or analysis and interpretation of data; took part in drafting the article or revising it critically for important intellectual content; agreed to submit to the current journal; gave final approval of the version to be published; and agreed to be accountable for all aspects of the work.

\section{Funding}

The Adelphi Migraine Disease-Specific Programme (DSP) was an independent survey conducted by Adelphi Real World. Lilly subscribed to the dataset from which this analysis and publication are derived. All authors had full access to all data and had final responsibility for the decision to submit for publication. Neither honoraria nor payments were made for authorship.

\section{Disclosure}

JF, RMN, WY, AT-H are employees and shareholders of Eli Lilly and Company, Indianapolis, IN, USA. SC and JJ are employees of Adelphi Real World, Bollington, UK. The authors report no other conflicts of interest in this work.

\section{References}

1. Headache Classification Committee of the International Headache Society. The International Classification of Headache Disorders, 3rd ed. Cephalalgia. 2018;38(1):1-211. doi:10.1177/0333102417738202

2. Lampl C, Thomas H, Stovner LJ, et al. Interictal burden attributable to episodic headache: findings from the Eurolight project. $J$ Headache Pain. 2016;17:9. doi:10.1186/s10194-016-0599-8

3. Woldeamanuel YW, Cowan RP. Migraine affects 1 in 10 people worldwide featuring recent rise: a systematic review and meta-analysis of community-based studies involving 6 million participants. $J$ Neurol Sci. 2017;15(372):307-315. doi:10.1016/j.jns.2016.11.071

4. Steiner TJ, Stovner LJ, Katsarava Z, et al. The impact of headache in Europe: principal results of the Eurolight project. J Headache Pain. 2014;15(1):31. doi:10.1186/1129-2377-15-31

5. Raggi A, Covelli V, Leonardi M, Grazzi L, Curone M, D’Amico D. Difficulties in work-related activities among migraineurs are scarcely collected: results from a literature review. Neurol Sci. 2014;35(Suppl 1):23-26. doi:10.1007/s10072-014-1736-2

6. Rencz F, Brodszky V, Péntek M, Bereczki D, Gulácsi L. Health state utilities for migraine based on attack frequency: a time trade-off study. Neurol Sci. 2015;36(2):197-202. doi:10.1007/s10072-014-1920-4

7. Buse DC, Lipton RB, Hallström Y, et al. Migraine-related disability, impact, and health-related quality of life among patients with episodic migraine receiving preventive treatment with erenumab. Cephalalgia. 2018;38(10):1622-1631. doi:10.1177/0333102418789072

8. Smitherman TA. Nonpharmacologic treatment of migraine. In: Smitherman TA, editor. Clinician's Manual on Migraine. Adis: Cham; 2016:69-77.

9. Hepp Z, Dodick DW, Varon SF, et al. Persistence and switching patterns of oral migraine prophylactic medications among patients with chronic migraine: a retrospective claims analysis. Cephalalgia. 2017;37(5):470-485. doi:10.1177/0333102416678382

10. Blumenfeld AM, Bloudek LM, Becker WJ, et al. Patterns of use and reasons for discontinuation of prophylactic medications for episodic migraine and chronic migraine: results from the second International Burden of Migraine Study (IBMS-II). Headache. 2013;53(4):644655. doi:10.1111/head.12055

11. Goadsby PJ, Schoenen J, Ferrari MD, Silberstein SD, Dodick D. Towards a definition of intractable headache for use in clinical practice and trials. Cephalalgia. 2006;26(9):1168-1170. doi:10.11 11/j.1468-2982.2006.01173.x

12. Silberstein SD. Practice parameter - evidence-based guidelines for migraine headache (an evidence-based review): report of the quality standards subcommittee of the American Academy of Neurology for the United States Headache Consortium. Neurology. 2000;55(6):754762. doi:10.1212/WNL.55.6.754

13. Hepp Z, Dodick DW, Varon SF, Gillard P, Hansen RN, Devine EB. Adherence to oral migraine-preventive medications among patients with chronic migraine. Cephalagia. 2015;35(6):478-488. doi:10.11 $77 / 0333102414547138$ 
14. Buse DC, Rupnow MF, Lipton RB. Assessing and managing all aspects of migraine: migraine attacks, migraine-related functional impairment, common comorbidities, and quality of life. Mayo Clin Proc. 2009;84(5):422-435. doi:10.1016/S0025-6196(11)60561-2

15. Martelletti P, Schwedt TJ, Lanteri-Minet M, et al. My Migraine Voice survey: a global study of disease burden among individuals with migraine for whom preventive treatments have failed. $J$ Headache Pain. 2018;19(1):115. doi:10.1186/s10194-018-0946-z

16. Anderson P, Benford M, Harris N, Karavali M, Piercy J. Real-world physician and patient behaviour across countries: Disease-Specific Programmes - a means to understand. Curr Med Res Opin. 2008;24 (11):3063-3072. doi:10.1185/03007990802457040

17. Higgins V, Piercy J, Roughley A, et al. Trends in medication use in patients with type 2 diabetes mellitus: a long-term view of real-world treatment between 2000 and 2015. Diabetes Metab Syndr Obes. 2016;9:371-380. doi:10.2147/DMSO.S120101

18. Babineaux SM, Curtis B, Holbrook T, et al. Evidence for validity of a national physician and patient-reported, cross-sectional survey in China and UK: the Disease Specific Programme. BMJ Open. 2016;6:e010352. doi:10.1136/bmjopen-2015-010352

19. Jhingran P, Osterhaus JT, Miller DW, Lee JT, Kirchdoerfer L. Development and validation of the Migraine-Specific Quality of Life Questionnaire. Headache. 1998;38(4):295-302. doi:10.1046/ j.1526-4610.1998.3804295.x

20. Stewart WF, Lipton RB, Kolodner K, Liberman J, Sawyer J. Reliability of the migraine disability assessment score in a population-based sample of headache sufferers. Cephalalgia. 1999;19 (2):107-114. doi:10.1046/j.1468-2982.1999.019002107.x

21. Stewart WF, Lipton RB, Dowson AJ, Sawyer J. Development and testing of the Migraine Disability Assessment (MIDAS) questionnaire to assess headache-related disability. Neurology. 2001;56(6 Suppl 1):S20-S28. doi:10.1212/WNL.56.suppl_1.S20

22. The EuroQol Group. EuroQol - a new facility for the measurement of health-related quality of life. Health Policy. 1990;16(3):199-208. doi:10.1016/0168-8510(90)90421-9

23. Brooks R. EuroQol: the current state of play. Health Policy. 1996;37 (1):53-72. doi:10.1016/0168-8510(96)00822-6

24. Reilly MC, Zbrozek AS, Dukes EM. The validity and reproducibility of a work productivity and activity impairment instrument. Pharmacoeconomics. 1993;4(5):353-365. doi:10.2165/00019053199304050-00006

25. National Institute for Health and Care Excellence (NICE). Position statement on use of the EQ-5D-5L valuation set for England; 2019. Available from: https://www.nice.org.uk/about/what-we-do/our-pro grammes/nice-guidance/technology-appraisal-guidance/eq-5d-5l. AccessedJune 15, 2021.

26. van Reenan M, Janssen B. EQ-5D-5L user guide. Basic information on how to use the EQ-5D-5L instrument; 2019. Available from: https:// euroqol.org/wp-content/uploads/2021/01/EQ-5D-5LUserguide-08-0421. pdf. Accessed June 15, 2021.

27. Szende A, Janssen B, Cabases J. Self-reported population health: an international perspective based on EQ-5D; 2014. Available from: https://ink.springer.com/content/pdf/10.1007\%2F978-94-007-75961.pdf. Accessed June 15, 2021.

ClinicoEconomics and Outcomes Research

\section{Publish your work in this journal}

ClinicoEconomics and Outcomes Research is an international, peerreviewed open-access journal focusing on Health Technology Assessment, Pharmacoeconomics and Outcomes Research in the areas of diagnosis, medical devices, and clinical, surgical and pharmacological intervention. The economic impact of health policy and health systems
28. Scher AI, Buse DC, Fanning KM, et al. Comorbid pain and migraine chronicity: the chronic migraine epidemiology and outcomes study. Neurology. 2017;89(5):461-468. doi:10.1212/WNL.0000000000004177

29. Giamberardino MA, Affaitati G, Martelletti P, et al. Impact of migraine on fibromyalgia symptoms. J Headache Pain. 2016;17:28. doi:10.1186/s10194-016-0619-8

30. Affaitati G, Costantini R, Tana C, Cipollone F, Giamberardino MA. Co-occurrence of pain syndromes. I Neural Transm (Vienna). 2020;127(4):625-646. doi:10.1007/s00702-019-02107-8

31. Pike J, Mutebi A, Shah N, et al. Factors associated with a history of failure and switching migraine prophylaxis treatment: an analysis of clinical practice data from the United States, Germany, France, and Japan. Value Health. 2016;19:A68 (abstr PND52). doi:10.1016/j. jval.2016.03.213

32. Sacco S, Bendtsen L, Ashina M, et al. European Headache Federation guideline on the use of monoclonal antibodies acting on the calcitonin gene related peptide or its receptor for migraine prevention. $J$ Headache Pain. 2019;20(1):6. doi:10.1186/s10194-018-0955-y

33. American Headache Society. The American Headache Society position statement on integrating new migraine treatments into clinical practice. Headache. 2019;59(1):1-18.

34. Abu Bakar N, Tanprawate S, Lambru G, Torkamani M, Jahanshahi M, Matharu M. Quality of life in primary headache disorders: a review. Cephalalgia. 2016;36(1):67-91. doi:10.1177/0333102415580099

35. Raub A, Chung P, Batra P, et al. Paid leave for personal illness: a detailed look at approaches across OECD countries; 2018. Available from https://www.worldpolicycenter.org/sites/default/files/WORLD $\%$ 20Report $\% 20-\% 20$ Personal $\% 20$ Medical $\% 20$ Leave $\% 20$ OECD $\%$ 20Country\%20Approaches_0.pdf. Accessed June 15, 2021.

36. Vo P, Fang J, Bilitou A, Laflamme AK, Gupta S. Patients' perspective on the burden of migraine in Europe: a cross-sectional analysis of survey data in France, Germany, Italy, Spain, and the United Kingdom. $J$ Headache Pain. 2018;19(1):82. doi:10.1186/s10194-018-0907-6

37. Shah N, Pike J, Mutebi A, et al. The use of migraine prophylaxis treatments in the United States of America: analysis of data from clinical practice. Headache. 2016;56(Supp11):abstr PS45.

38. Shah N, Pike J, Mutebi A, et al. Characterizing patient-reported health-related quality of life measures among users and non-users of migraine prophylaxis treatment: an analysis of clinical practice data from the United States, Germany, France, and Japan. Value Health. 2016;19:A68 (poster PND53). doi:10.1016/j.jval.2016.03.214

39. Katsarava Z, Mania M, Lampl C, Herberhold J, Steiner TJ. Poor medical care for people with migraine in Europe - evidence from the Eurolight study. J Headache Pain. 2018;27(9):1000-1004.

40. Dodick DW, Loder EW, Manack Adams A, et al. Assessing barriers to chronic migraine consultation, diagnosis, and treatment: results from the Chronic Migraine Epidemiology and Outcomes (CaMEO) study. Headache. 2016;56(5):821-834. doi:10.1111/head.12774

\section{Dovepress}

organization also constitute important areas of coverage. The manuscript management system is completely online and includes a very quick and fair peer-review system, which is all easy to use. Visit http://www.dovepress.com/testimonials.php to read real quotes from published authors. 